\title{
Signal Analysis for High Speed Acoustic Data Using Acoustic Emission Analysis Tool
}

\author{
Shivani .G ${ }^{1}$, Vandna . $\mathbf{B}^{2}$ \\ ${ }^{1}$ PG Scholar, Department of ECE, SDDIET-Panchkula, Barwala, India \\ ${ }^{2}$ Assistant Professor, Department of ECE, SDDIET-Panchkula, Barwala, India
}

\begin{abstract}
Acoustic Emission (AE) refers to the generation of transient elastic waves produced by a sudden redistribution of stress in a material. In this work, AE analysis tool is utilized to process high speed AE data. In the whole work, simplification of raw data detected using different $A E$ sensors and then filtering of the data is done with the help of AE Analyzing tool and after that different signal analyzing functions/algorithms (FFT, STFT and its spectrogram) on MATLAB is applied. The AE has analytical functions like Total, Maximum, Minimum and Average. The millions of the data point corresponding to micro-second scale of AE data are simplified into thousands or hundreds of data points (by opting second-wise, minute-wise or hourly data) by using this AE tool and thereafter it can be analyzed efficiently and speedily.
\end{abstract}

Keywords: Acoustic Emission (AE), Fast Fourier Transform (FFT), Short time Fourier Transform (STFT), Universal Testing Machine (UTM).

\section{Introduction}

Acoustic Emission (AE) refers to the generation of transient elastic waves produced by a sudden redistribution of stress in a material. When a structure is subjected to an external stimulus (change in pressure, load, or temperature), localized sources trigger the release of energy, in the form of stress waves, which propagate to the surface and are recorded by sensors. With the right equipment and setup, motions on the order of picometers $\left(10^{-12} \mathrm{~m}\right)$ can be identified. Sources of $\mathrm{AE}$ may vary from natural events like earthquakes and rockbursts to the initiation and growth of cracks, slip and dislocation movements, melting, twinning, and phase transformations in metals. In composites, matrix cracking and fiber breakage and de-bonding contribute to acoustic emissions. $\mathrm{AE}^{\text {es }} \mathrm{s}$ have also been measured and recorded in polymers, wood, and concrete, among other materials. Detection and analysis of AE signals can supply valuable information regarding the origin and importance of a discontinuity in a material. Because of the versatility of Acoustic Emission Testing (AET), it has many industrial applications (e.g. assessing structural integrity, detecting flaws, testing for leaks, or monitoring weld quality) and is used extensively as a research tool. AE sensors are piezoelectric crystals that convert displacement (a variation of pressure) into an electrical voltage. The sensors must all have an identical response and they should be calibrated. They are normally held in place using metallic clamps for steel structures or bonded to concrete. These are connected to the $\mathrm{AE}$ system using coaxial cables with shielding to prevent electro-magnetic interference. A resonant frequency of $30 \mathrm{kHz}-100 \mathrm{kHz}$ is typical for concrete applications; whereas $100 \mathrm{kHz}$ and $200 \mathrm{kHz}$ are used for metallic structures. Higher frequency sensors can be used in high noise environments but only for local monitoring due to the higher attenuation at these frequencies. A typical AE system comprises a high speed Digital Signal Processor (DSP),

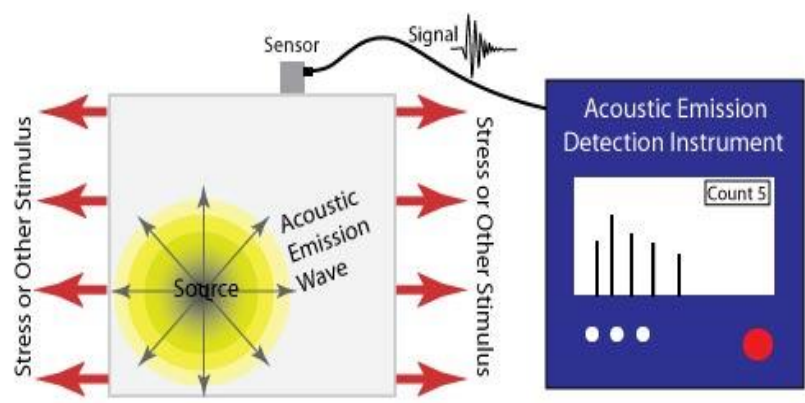

Figure 1: A Conceptual Acoustic Emission System

$\mathrm{AE}$ processing boards with individual processing channels for each sensor (i.e. a non multiplexing system) and the ability to program the settings for signal thresholds and frequency range to enable the $\mathrm{AE}$ signal to be filtered. The following $\mathrm{AE}$ sensors are being used.

\begin{tabular}{|c|c|c|c|c|}
\hline Sensor type & $\begin{array}{c}\text { Operating } \\
\text { frequency } \\
\text { range(in } \\
\text { KHz) }\end{array}$ & $\begin{array}{c}\text { Resonant } \\
\text { frequency }\end{array}$ & $\begin{array}{c}\text { Peak } \\
\text { sensitivity }\end{array}$ & $\begin{array}{c}\text { Operating } \\
\text { Temp. range } \\
\text { (in degree } \\
\text { Celsius) }\end{array}$ \\
\hline AE1(R.45I) & $1-30$ & $20 \mathrm{kHz}$ & $124 \mathrm{~dB}$ & $-35-75$ \\
\hline AE2(R1.5I) & $5-20$ & $14 \mathrm{kHz}$ & $124 \mathrm{~dB}$ & $-35-75$ \\
\hline AE3(R3I-AST) & $10-70$ & $31 \mathrm{kHz}$ & $121 \mathrm{~dB}$ & $-35-75$ \\
\hline AE4(R6I-AST) & $40-100$ & $98 \mathrm{kHz}$ & $117 \mathrm{~dB}$ & $-35-75$ \\
\hline $\begin{array}{c}\text { AE5(R15I- } \\
\text { AST) }\end{array}$ & $50-200$ & $150 \mathrm{kHz}$ & $109 \mathrm{~dB}$ & $-35-75$ \\
\hline $\begin{array}{c}\text { AE6(R30I- } \\
\text { AST) }\end{array}$ & $125-450$ & $350 \mathrm{kHz}$ & $97 \mathrm{~dB}$ & $-35-75$ \\
\hline AE7(R3 $\alpha)$ & $25-530$ & $29 \mathrm{kHz}$ & $80 \mathrm{~dB}$ & $-65-75$ \\
\hline
\end{tabular}




\section{International Journal of Science and Research (IJSR) \\ ISSN (Online): 2319-7064}

Index Copernicus Value (2013): 6.14 | Impact Factor (2014): 5.611

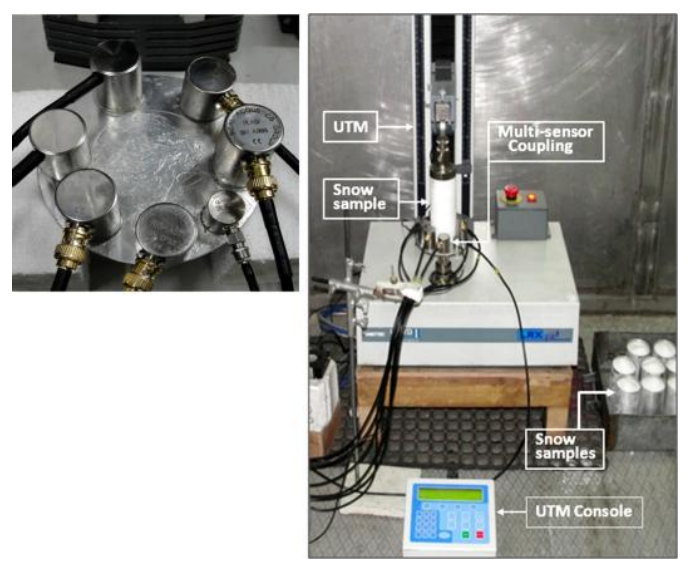

Figure 2: (a) AE multi-sensor coupling using circular arrestor base. (b) Experimental setup of multi-channel acoustic emission system and test apparatus (Universal Testing Machine: UTM) installed inside the cold laboratory $\left(-40^{\circ} \mathrm{C}\right)$.

For the detection of a common AE activity, simultaneous by all the sensors, a multi sensor coupling approach can be useful for assessing the relative performance of several types of AE sensors. A multi-sensor coupler was made from a circular plate of aluminum (diameter $0.14 \mathrm{~m}$ and thickness $0.003 \mathrm{~m}$ ), as shown in Fig. 1.3. For suppression of external vibrations and other interferences caused by the mechanical system of the uni-axial compression and tension apparatus (Universal Testing Machine: UTM) and of the cold laboratory, an insulation pad had been provided below the arrestor plate. For the acquisition of the AE activity, a multichannel acoustic emission system (16-channel Digital Acoustic Emission system, Micro II SAMOS, supplied by Physical Acoustics Corporation, USA)with an operating frequency range from $1 \mathrm{kHz}$ to $400 \mathrm{kHz}$ was used.

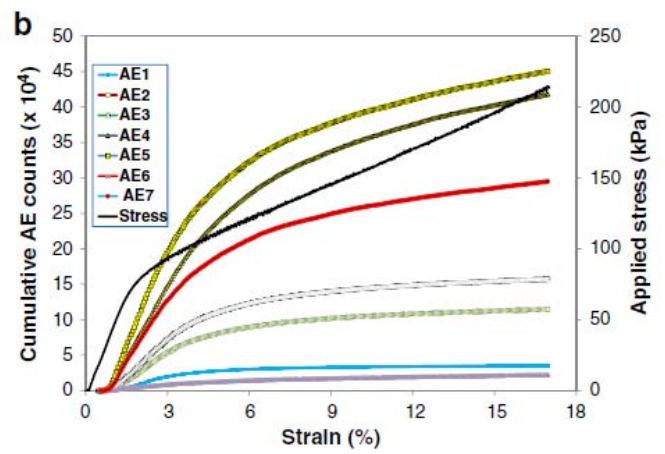

Figure 3: Graph of cumulative AE counts Vs Applied stress

The cumulative AE counts detected by the sensors AE 5 (50 $\mathrm{kHz}-200 \mathrm{kHz}$ ) were highest among all followed by sensor AE $4(40 \mathrm{kHz}-100 \mathrm{kHz})$ which had slightly lower performance. Also, the sensor AE 7 performed well in a wide frequency range of $25 \mathrm{kHz}$ to $530 \mathrm{kHz}$ which can be useful especially for wide band field applications in snow. The results suggest that the emitted frequency spectrum is in the frequency range of $40 \mathrm{kHz}$ to $200 \mathrm{kHz}$. Furthermore, Fig. 3 reveals that there is a good correlation between the cumulative AE counts and the applied stress as measured by the load cell.

\section{Literature Survey}

J.C. Kapil, P. Datt et al. [1] [2015] proposed Acoustic emission characteristics and b-value estimate in relation to waveform analysis for damage response of snow. The AE setup consists of a high speed data acquisition system with sixteen input channels having a bandwidth of $400 \mathrm{kHz}$ and allowing sampling rates of up to 1 MSPS for each channel. J.C. Kapil, P. Datt, A. Kumar, P.K Satyawali et al. [2] (2014) proposed the multi-sensor couplers and waveguides for efficient detection for acoustic emission behavior of snow. G. Michlmayr, D. Cohen et al [9] (2012) proposed the sources and characteristics of acoustic emissions from mechanically stressed geologic granular media and for geological materials is also implemented for snow damage behavior. D. Amitrano [7] (2012) proposed the variability in the power-law distributions of rupture events, The European Physical Journal-Special Topics. A power law was established for a particular set of data points deduced from a particular time window of the time series amplitude distribution. Weiss, M. Weavers et al [32] (2000) proposed the waveform based analysis techniques for the reliable acoustic emission composite structures. The brittle fracturing in snow caused through visco-plastic deformation is analogues to a critical phenomenon and it may occur in burst of relaxation following a power law for energy. W.F. Lawrence [45] (1980) proposed the Acoustic Emission response of snow. The acoustic emissions in snow are continuous and burst types of stress waves which are directly related to its deformation and failure mechanism induced through cleavage of inter-granular bonds, crack initiation, crack extension, dislocation movements and plastic deformation along with creep-glide processes occurring in a slope. W.F. Lawrence, T.R. Williams [50] (1976) proposed the seismic signals associated with avalanches. Characteristic AE signals were recorded by them from different types of avalanches through a broadband acoustical survey of snow avalanches.

\section{Methods / Approach}

Filtering /windowing of High speed Acoustic Emission data with the help of AE tool. Displaying processed data. Applying different signal analysis using functions / algorithms with MATLAB i.e. FFT (Fast Fourier Transform), ST-FT (Short time FT) and its spectrogram. The main objective is simplification of high speed AE data ( $\mu \mathrm{sec}$ scale) into analogous lowest speed AE data (sec scale) using Java based AE Analysis tool for high speed real time data monitoring of any failure activity using AE activity, deriving the optimum frequency and signal strength information corresponding to different failure activities. AE activity of deformation of snow in terms of FFT, STFT and its spectrogram.

\section{Results and Discussion}

Analysis of the various sensors was carried out however in this case only and the result of one of the sensor 3 (Sensor AE3, R3I-AST) is shown below. Channel 3 is attached to the sensor R3I-AST. Channel 3 operates in the frequency range 


\section{International Journal of Science and Research (IJSR) \\ ISSN (Online): 2319-7064 \\ Index Copernicus Value (2013): 6.14 | Impact Factor (2014): 5.611}

$(10 \mathrm{kHz}-70 \mathrm{kHz})$. It has the resonant frequency of $31 \mathrm{kHz}$ and peak sensitivity $121 \mathrm{~dB}$.

\section{(a) Average Second Wise}

FFT of Average Second wise is shown in fig. 4. It reveals the magnitude $(\mathrm{dB})$ and phase response in (radian) with respect to the normalized frequency $(\mathrm{kHz}$ i.e. sampling frequency is $1000 \mathrm{~Hz}$ in the half cycle).

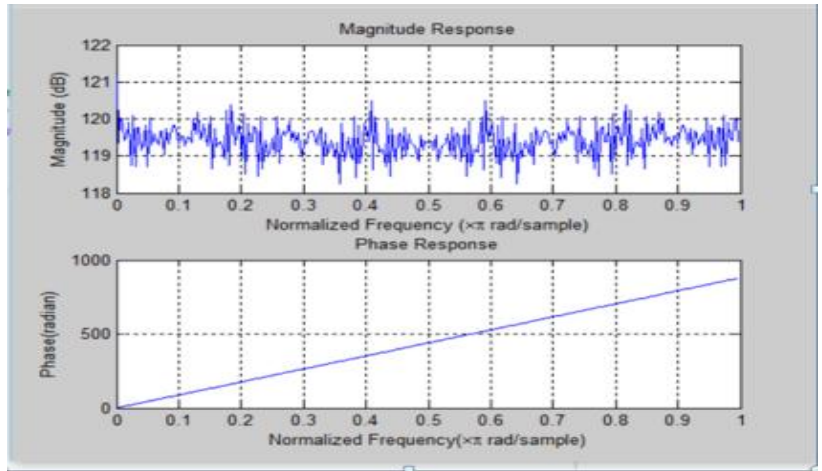

Figure 4: FFT

This diagram shows that the magnitude is approximately $120.3 \mathrm{~dB}$ in low side frequency i.e. $50 \mathrm{kHz}$. The peak frequency is at $210 \mathrm{kHz}$ and at $280 \mathrm{kHz}$. Since this sensor has a peak sensitivity of $121 \mathrm{~dB}$ and its magnitude below it. This is because as the bandwidth is the difference between 2 frequencies which gives us the peak values but there are frequencies lying below bandwidth. So, when there is no peak frequency, channel will respond to that frequency which is present below $3 \mathrm{~dB}$. This waveform is the weak signal and mostly contains noise. Reverberation is being formed in this part. So due to noise, the phase response is also uniform.

STFT is shown in fig. 5. It reveals the frequency (in $\mathrm{kHz}$ ) with respect to time $(\mathrm{x} 1000 \mathrm{~s})$.

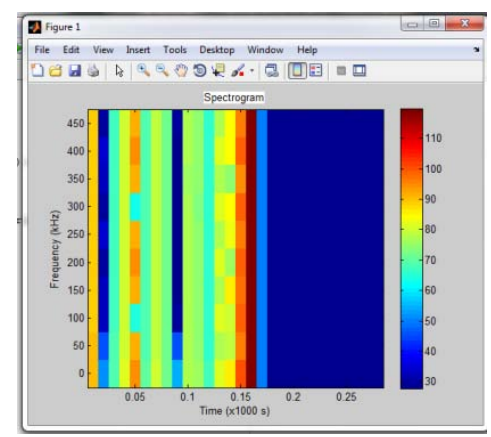

Figure 5: STFT and spectrogram

This diagram shows that the process is occurring for 170 seconds. Further after that, significant frequency information is not derived. It shows the region of frequency between ( 0 $500 \mathrm{kHz}$ ) for $155-160 \mathrm{sec}$ is most dominant. It means that this region is the region for failure indicating that some disaster is going to occur. Other color bands are less dominant.

\section{(b) Maximum Second Wise}

FFT of maximum second wise is shown in fig. 6. It reveals the magnitude response and phase response with respect to the normalized frequency.

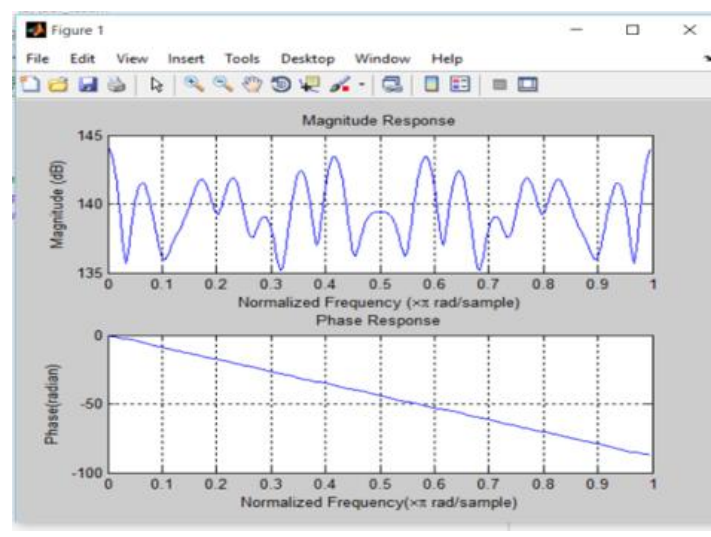

Figure 6: FFT

STFT is shown in fig. 7. It reveals the frequency Vs Time graph.

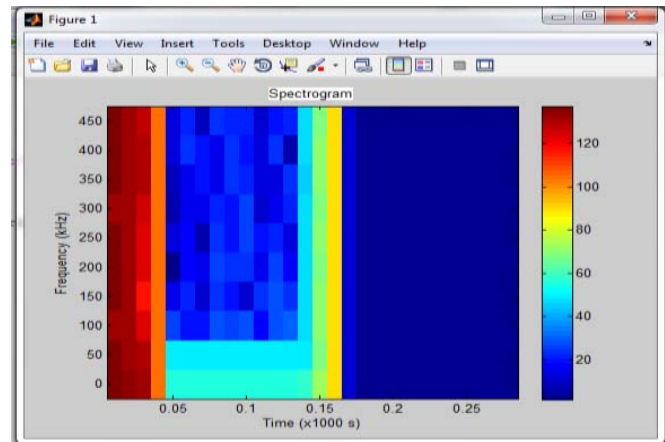

Figure 7: STFT and spectrogram

This diagram shows the frequency strip between $0-450 \mathrm{kHz}$ for $30 \mathrm{sec}$ is of maximum magnitude and is most dominant. The useful region lies till $180 \mathrm{sec}$. Further after this significant information is not derived.

\section{(c) Minimum Second Wise}

FFT of minimum second wise is shown in fig. 8. It describes the magnitude and phase response with respect to the normalized frequency.

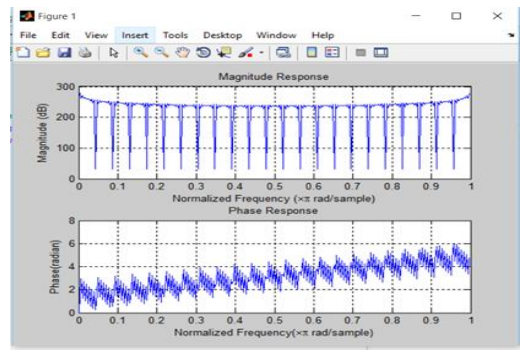

Figure 8: FFT

This diagram shows the minimum value of RMS parameter corresponding to maximum magnitude of $280 \mathrm{~dB}$ of frequencies less than $20 \mathrm{kHz}$ and more than $480 \mathrm{kHz}$. The rest frequency components tell us the minimum values between $(20 \mathrm{kHz}-480 \mathrm{kHz})$. Due to distortion, phase reversal is taking place and is of increasing phase with respect to frequency.

STFT of minimum second wise is shown in fig. 9. It describes the frequency component with respect to the time. 


\section{International Journal of Science and Research (IJSR) \\ ISSN (Online): 2319-7064}

Index Copernicus Value (2013): 6.14 | Impact Factor (2014): 5.611

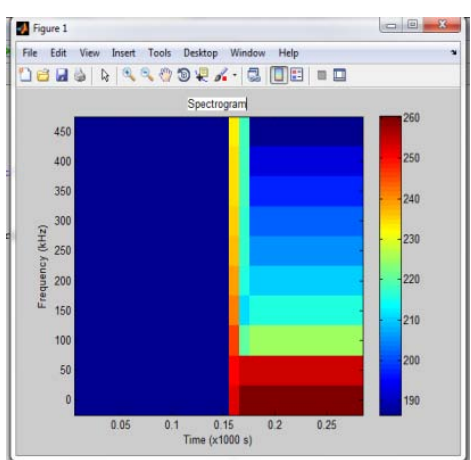

Figure 9: STFT and spectrogram

This diagram shows that till $150 \mathrm{sec}$, further significant information is not derived. The most dominant region is of $30 \mathrm{kHz}$ for $150 \mathrm{sec}$ duration $(150-300 \mathrm{sec})$. Rest region is less prominent.

Unprocessed data of the RMS parameter (Excel file) contains a lot of noise and distortion. It does not depict clear information. So processed data with the help of Acoustic Emission Analyzer, data is filtered and channels are attached to the sensors which cannot be done in unprocessed data. There is no provision of these AE parameters and analytical functions like Average, Maximum, Minimum and Total second wise value. Further, FFT and STFT and its spectrogram have been done. FFT is easier and is in frequency domain. It is not precise and does not give any information about time domain. So, STFT is being used which is a time-frequency information, although time window is short. The advantage of this is frequency resolution is good and is more useful to monitor natural hazard activities producing particular frequency range. However, wavelet Transform could also be used but its frequency resolution is not good as time resolution is good. A mother wavelet superimposes the original signal, thus pseudo signal is generated, so frequency information is not true. Our main motto is to generate frequency.

\section{Conclusion}

Snow samples were taken and the raw data was collected and with the help of ,Acoustic Emission Analyzing toole data was filtered and huge amount of data was transformed into smaller periodic wise data. As the data in large memory of GB order which is difficult to process in a single file would be processed easily using this tool. So, this tool monitors and interprets in micro-seconds or nano-seconds. Data is not changed but it is the monitoring which gives the information. This tool stores the data and transmits it easily. With the help of AE Analyzing tool, data is analyzed. Different sensors perform different information. Sensors 3, 4 and 7 have been studied in this work. Average, Maximum and Minimum of second wise data for different channels/sensors of FFT, STFT and spectrogram of RMS parameter have been performed. FFT is easier but not precise as it gives only frequency domain information. So, to overcome this STFT was performed which yields a time frequency information although its time window is short. In this time resolution is not good but frequency resolution is good. So, this is more useful to monitor natural hazards producing particular frequency band range. Besides this, wavelet transform could also be used but its time resolution is good and frequency resolution is not good, thus pseudo signal could have been generated. So, wavelet transform has been discarded as our main motive is to generate frequency. From the above observations, we found that in sensor (AE3) channel is distorted and in sensor 4, channel is distinct and less distorted and gives a clear information and in sensor 7 , channel is least distorted. Magnitude of FFT varies according to these sensors and a good phase response is received. Its spectrogram tells us that the highest magnitude of the color bar is the warning signal of any disaster or occurrence.

\section{Future Scope}

The high speed analysis tool is of wide applications to extract the useful information from various natural processes such as failure of a structure or a slope. Hence for real time monitoring of any such event can be immediately processed using such tools and warning can be generated real time also. For real time operational avalanche warning this tool would be applied by defence and research institutes.

\section{References}

[1] Kapil J.C., Datt P. Acoustic emission characteristics and b-value estimate in relation to waveform analysis for damage response of snow, 2015

[2] Kapil J.C., Datt P., Kumar A., Singh K., Kumar V. and Satyawali P.K.Multi-sensor couplers and waveguides for efficient detection of acoustic emission behavior of snow. Cold Reg. sci. \& Technol, 2014

[3] Amitrano D. Variability in the power-law distributions of rupture events, The European Physical Journal-Special Topics, 2012

[4] Michlmayr G., Cohen D., Dani Or. Sources and characteristics of acoustic emissions from mechanically stressed geologic granular media, 2012

[5] Weiss, Surgeon M., Buelens C., Wevers M. and Meester P.D. "Waveform based analysis techniques for the reliable acoustic emission composite structures", Journal of Acoustic Emission, 2000

[6] Lawrence W.F. The Acoustic Emission response of snow. J. Glaciol, 1980

[7] St. Lawrenc W.F., Williams T.R. Seismic signals associated with avalanches. J. Glaciol, 1976 